\title{
Über das Cerebron.
}

\author{
IV. Mitteilung.
}

Von

Hermann Loening und H. Thierfelder.

(Aus dem physiologisch-chemischen Institut der Universität Tübingen.)

(Der Redaktion zugegangen am 10. August 1910.)

Untereinander nicht übereinstimmende Beobachtungen ${ }^{1)}$ bei der Untersuchung des basischen Anteils, welcher bei der Spaltung von Cerebronpräparaten verschiedener Darstellung erhalten wurde, ließen es zweckmäßig erscheinen, das Cerebron nochmals auf seine Reinheit zu prüfen. Bei diesen Untersuchungen begegneten wir einer dem Cerebron sehr ähnlichenund schwer von ihm abtrennbaren Substanz, welche bei den früheren Darstellungen nicht immer völlig entfernt worden sein dürfte.

Die Erkennung einer nicht zu kleinen Beimengung dieses Körpers in Cerebronpräparaten wird ermöglicht durch die von uns aufgefundene Eigenschaft des Cerebrons, sich in kürzester Zeit in prächtige Krystalle zu verwandeln, wenn man es mit Methylalkohol oder 10\% Chloroform enthaltenden Methylalkohol in einer Menge, welche auch beim Sieden zur Lösung nicht hinreicht, übergießt und auf dem Wasserbad zum Kochen erhitzt. Die Krystallformen gleichen vollkommen denen, welche allmählich aus in 85\% igem Alkohol suspendiertem Cerebron bei etwa $50^{\circ}$ entstehen. ${ }^{2}$ ) Diese Eigenschaft zeigt der im übrigen dem Cerebron sich sehr ähnlich verhaltende Körper nicht, er bleibt amorph. In dieser Prüfung, welche sich schon mit ganz kleinen Mengen Substanz ausführen läßt und am besten in einem S. 286.

1) F. Kitagawa und H. Thierfelder, Diese Zeitschrift, Bd. XLIX,

1) Emil Wörner und H. Thierfelder, Diese Zeitschrift, Bd. XXX, S. 542 . 
kleinen, mit Steigrohr versehenen Rundkölbchen vorgenommen wird, haben wir ein bequemes Mittel, die fortschreitende Reinheit des Cerebrons zu kontrollieren.

Als Ausgangsmaterial diente bei dieser Untersuchung Rindergehirn. Die Verarbeitung geschah in etwas anderer Weise wie bisher. Die von Membranen und Blut befreite und fein zerhackte Gehirnmasse wurde auf Glasplatten in dünner Schicht aufgetragen. Die Platten lagen auf einer mit dicker Sandschicht bedeckten Eisenplatte und wurden durch untergestellte Brenner so erwärmt, daß die Temperatur des Sandes an keiner Stelle $50-53^{\circ}$ überstieg. Ein über den Platten angebrachter Flügelventilator sorgte für rasche Erneuerung der Luft. In den ersten 3 Stunden wurde die Masse etwa alle 10 Minuten mit einem Spatel gewendet, dann seltener. Nach 10 Stunden hatte sie eine Wassermenge, welche bei verschiedenen Darstellungen zwischen 72 und $75 \%$ des Gewichts des frischen Gehirns schwankte, abgegeben und eine helle Fleischfarbe angenommen. Sie verblieb nun noch längere Zeit im Exsikkator und wurde darauf im Soxhletschen Extraktionsapparat mit Äther ausgezogen und zwar unter zweimaligem Wechsel des Äthers je 3-4 Stunden. Die in den ätherischen Lösungen erfolgenden Abscheidungen trennten wir in der Zentrifuge ab, zerteilten den Bodensatz in frischem Äther, zentrifugierten wieder und wiederholten die Behandlung nochmals für den Fall, daß der Äther noch gefärbt war.

Die so erhaltene weiße Masse verarbeiteten wir auf Cerebron. Das unendlich mühsame Verfahren soll nicht in allen Einzelheiten, sondern nur in seinen Grundzügen angegeben werden.

Zunächst erfolgte ein sechsmal wiederholtes Umlösen aus 75\% Chloroform enthaltendem Methylalkohol. Damit erreichten wir vor allem eine Entfernung des größten Teiles der phosphorhaltigen Bestandteile, aber auch schon eine gewisse Trennung der krystallisierenden Substanz (Cerebron) von der amorphen, welch letztere der Hauptmenge nach in den Mutterlaugen blieb und sich nach deren Einengen auf ein kleines Volumen zusammen mit krystallisierender Substanz abschied. 
Beide Teile (der durch Umlösen gewonnene, welcher vorwiegend aus Cerebron bestand, und der aus den eingeengten Mutterlaugen erhaltene, in welchem sich neben amorpher auch reichliche Mengen krystallisierender Substanz befanden) wurden nun, jeder für sich, in großen Mengen heißem 20\% Chloroform enthaltendem Methylalkohol gelöst und die beim Abkühlen innerhalb gewisser Temperaturgrenzen erfolgenden Abscheidungen mittels Filtrierens durch Warmwassertrichter von einander getrennt. Jede dieser so erhaltenen Fraktionen prüften wir in kleinen Proben in der oben beschriebenen Weise auf Krystallisierbarkeit (ob völlige Umwandlung erfolgte oder reichliche oder spärliche, oder ob die Krystallisation völlig ausblieb) und vereinigten die sich gleich oder ähnlich verhaltenden. Die zuerst ausgeschiedenen Anteile zeigten die reichlichste Krystallisation, die zuletzt ausgefallenen blieben bei dieser Prüfung amorph. Eine weitere Trennung innerhalb der so gewonnenen einzelnen Fraktionen ließ sich durch wiederholte Extraktion mit 10\% Chloroform enthaltendem Methylalkohol und weiterhin mit Methylalkohol bei $50^{\circ}$ erzielen. Die beim Erkalten der Extraktionsflüssigkeiten erfolgenden Abscheidungen wurden abfiltriert und ebenso wie die extrahierten Massen wie oben geprüft und mit den ein gleiches Verhalten zeigenden zusammengetan. Die extrahierten Substanzen wurden mit der Zahl der Extraktionen immer ärmer an dem amorphen Körper, die ersten Extraktionsflüssigkeiten waren relativ am reichsten an ihm.

Übrigens wurde wiederholt beobachtet, daß eine durchaus keine Krystallbildung zeigende Portion nach wiederholter Extraktion bei $50^{\circ}$ doch noch Krystalle lieferte und daß umgekehrt einer andern, welche völlig krystallisiert erschien, doch noch amorphe Anteile durch Ausziehen mit Chloroformmethylalkohol oder Methylalkohol bei $50^{\circ}$ entzogen werden konnten. Eine sichere Entscheidung gibt also die Probe allein nicht, wenn es sich um geringe Beimengungen handelt.

Auf dem beschriebenen Weg wurden schließlich vier Fraktionen erhalten, von denen die eine (D) völlig krystallisierte, eine zweite (A) ganz amorph blieb, während von den zwischen- 
liegenden beiden, welche Mischungen darstellten, die eine (C) reicher an krystallisierender Substanz war als die andere (B). Die Fraktion D, welche für reines Cerebron gehalten wurde, zerlegten wir nun nochmals in drei Unterfraktionen und zwar in der Weise, daß wir sie zunächst zweimal mit Methylalkohol bei $50^{\circ}$ auszogen und die beim Erkalten erfolgenden Abscheidungen abfiltrierten und vereinigten $\left(D^{1}\right)$. Die ungelöste Hauptmenge wurde in heißem Methylalkohol gelöst. Die unter beständigem Schütteln beim langsamen Erkalten bis auf etwa $40^{\circ}$ erfolgende Abscheidung, welche durch Warmwassertrichter abfiltriert wurde, stellte die Unterfraktion $D^{3}$ dar und das beim völligen Erkalten des Filtrats Ausfallende die Unterfraktion $D^{2}$. Diese drei Unterfraktionen wurden untereinander und mit der Fraktion A (teilweise auch mit der Fraktion B) verglichen. Während $D^{2}$ und $D^{3}$ sich beim Erhitzen mit Methylalkohol sofort und vollständig in Krystalle verwandelten, war bei $D^{1}$ die Umwandlung keine ganz vollständige. Zwischen den Krystallen ließen sich noch vereinzelte amorphe Gebilde erkennen. Daß diese Fraktion noch nicht ganz reines Cerebron darstellte, ergab sich auch daraus, daß ihre Lösung in $10 \%$ Schwefelsäure enthaltendem Methylalkohol bei mehrstündigem Erhitzen einen, allerdings nur ganz leichten, gelblichen Farbenton (er war nur beim Vergleich mit Wasser sicher zu erkennen) annahm, eine Eigenschaft, welche die Fraktion $\mathrm{A}$ in ausgesprochener Weise zeigte, die den Fraktionen $\mathrm{D}^{2}$ und $\mathrm{D}^{3}$ aber ganz fehlte. Diese beiden blieben bei 3 stündigem Kochen mit der genannten Flüssigkeit farblos. Die Beimengung kann aber anderseits eine nur ganz geringe sein. Das geht auch daraus hervor, daß die Fraktion $D^{1}$ dieselbe Löslichkeit in Methylalkohol wie $\mathrm{D}^{2}$ und $\mathrm{D}^{3}$ zeigte, während die Fraktion $\mathrm{A}$ wesentlich löslicher war und die Löslichkeit der Fraktion B dazwischen lag.

Für diese Löslichkeitsbestimmungen brachten wir je $0,1 \mathrm{~g}$ Substanz mit $30 \mathrm{ccm}$ Methylalkohol in Kölbchen von gleicher Größe und Form, lösten sie bei aufgesetztem Steigrohr im Wasserbad, nahmen die Kölbchen, sobald die Flüssigkeit eben ins Sieden gekommen war, heraus und notierten die Zeit, welche bis zur beginnenden Ausscheidung, sowie die, welche bis zur vollendeten 
Ausscheidung, d. h. bis zur Verwandlung der ganzen Flüssigkeit in eine Gallerte, verstrich. Nachdem die Kölbchen bis zum nächsten Tage verschlossen stehen geblieben waren, zerteilten wir die Gallerte durch starkes Schütteln, filtrierten und bestimmten in je $20 \mathrm{ccm}$ des Filtrats den Trockenrückstand. Die erhaltenen Werte sind in folgender Tabelle verzeichnet.

\begin{tabular}{|c|c|c|c|c|}
\hline \multirow{2}{*}{ Fraktion } & Beginn & Ende & \multicolumn{2}{|c|}{ Trockenrückstand in } \\
\hline & der Aus & heidung & $\begin{array}{c}20 \mathrm{ccm} \\
\mathrm{g}\end{array}$ & $\begin{array}{c}100 \mathrm{ccm} \\
\mathrm{g}\end{array}$ \\
\hline $\mathbf{A}$ & nach 13 Min. & nach 24 Min. & 0,0051 & 0,0255 \\
\hline B & $\gg 10^{1 / 2} \gg$ & $>19^{1 / 2}$ & 0,0036 & 0,0180 \\
\hline$D^{1}$ & $\gg \quad 7^{1} / 4 \gg$ & $\triangleright 15$ & 0,0025 & 0,0125 \\
\hline $\mathrm{D}^{2}$ & $\gg 8$ & $\gg 14^{3} / 4 \gg$ & 0,0025 & 0,0125 \\
\hline $\mathrm{D}^{3}$ & 8 & $\triangleright 14^{3} / 4 \gg$ & 0,0027 & 0,0135 \\
\hline
\end{tabular}

Die Substanz A löste sich auch leichter in heißem Methylalkohol, als die andern. Die Elementaranalyse ergab keine Unterschiede, wie folgende Zusammenstellung zeigt.

Fraktion A.

1. $0,1390 \mathrm{~g}$ Substanz liefern $0,3557 \mathrm{~g} \mathrm{CO}_{2}$ und $0,1400 \mathrm{~g} \mathrm{H}_{\mathbf{2}} \mathrm{O}$, das ist $69,79 \% \mathrm{C}$ und $11,19 \% \mathrm{H}$.

2. $0,1643 \mathrm{~g}$ Substanz liefern $0,4208 \mathrm{~g} \mathrm{CO}_{2}$ und $0,1673 \mathrm{~g} \mathrm{H}_{2} \mathrm{O}$, das ist $69,85 \% \mathrm{C}$ und $11,31 \% \mathrm{H}$. $1,63 \% \mathrm{~N}$.

3. $0,4887 \mathrm{~g}$ Substanz verbrauchen $5,70 \mathrm{ccm} \mathrm{n} / 10$-Säure, das ist Fraktion $D^{1}$.

1. $0,1553 \mathrm{~g}$ Substanz liefern $0,3969 \mathrm{~g} \mathrm{CO}_{\mathrm{z}}$ und $0,1595 \mathrm{~g} \mathrm{H}_{\mathrm{z}} \mathrm{O}$, das ist $69,70 \% \mathrm{C}$ und $11,41 \% \mathrm{H}$.

2. $0,1272 \mathrm{~g}$ Substanz liefern $0,3260 \mathrm{~g} \mathrm{CO}_{2}$ und $0,1306 \mathrm{~g} \mathrm{H}_{2} \mathrm{O}$, das ist $69,90 \% \mathrm{C}$ und $11,41 \% \mathrm{H}$.

3. $0,2731 \mathrm{~g}$ Substanz verbrauchen $3,22 \mathrm{ccm} \mathrm{n} / 10$-Säure, das ist $1,65 \% \mathrm{~N}$.

\section{Fraktion $D^{2}$.}

1. $0,1791 \mathrm{~g}$ Substanz liefern $0,4579 \mathrm{~g} \mathrm{CO}_{2}$ und $0,1810 \mathrm{~g} \mathrm{H}_{2} \mathrm{O}$, das ist $69,73 \% \mathrm{C}$ und $11,23 \% \mathrm{H}$.

2. $0,1001 \mathrm{~g}$ Substanz liefern $0,2572 \mathrm{~g} \mathrm{CO}_{2}$ und $0,1009 \mathrm{~g} \mathrm{H}_{2} \mathrm{O}$, das ist $70,08 \% \mathrm{C}$ und $11,20 \% \mathrm{H}$.

3. $0,2975 \mathrm{~g}$ Substanz verbrauchen $3,65 \mathrm{ccm} \mathrm{n} / 10$-Säure, das ist $1,72 \% \mathrm{~N}$. 
Fraktion $D^{3}$.

1. $0,1260 \mathrm{~g}$ Substanz liefern $0,3240 \mathrm{~g} \mathrm{CO}_{2}$ und $0,1272 \mathrm{~g} \mathrm{H}_{2} \mathrm{O}$, das ist $70,13 \% \mathrm{C}$ und $11,22 \% \mathrm{H}$.

2. $0,1119 \mathrm{~g}$ Substanz liefern $0,2879 \mathrm{~g} \mathrm{CO}_{2}$ und $0,1146 \mathrm{~g} \mathrm{H}_{2} \mathrm{O}$, das ist $70,17 \% \mathrm{C}$ und $11,38 \% \mathrm{H}$.

3. $0,2225 \mathrm{~g}$ Substanz verbrauchen $2,75 \mathrm{ccm} \mathrm{n} / 10-S a ̈ u r e$, das ist $1,73 \% \mathrm{~N}$.

\begin{tabular}{c|r|r|r|r|r|r|r|r}
\hline \hline & \multicolumn{2}{|c|}{ Fraktion A } & \multicolumn{2}{|c|}{ Fraktion $D^{1}$} & \multicolumn{2}{|c|}{ Fraktion D2 } & \multicolumn{2}{c}{ Fraktion $D^{3}$} \\
\hline C & 69,79 & 69,85 & 69,70 & 69,90 & 69,73 & 70,08 & 70,13 & 70,17 \\
H & 11,19 & 11,31 & 11,41 & 11,41 & 11,23 & 11,20 & 11,22 & 11,38 \\
N & 1,63 & - & 1,65 & - & 1,72 & - & 1,73 & -
\end{tabular}

Es muß auffallend erscheinen, daß die Werte für Kohlenstoff durchgehend höher sind, als die früher für Cerebron erhaltenen, deren Durchschnitt $69,19^{\circ} \%$ beträgt. ${ }^{1}$ ) Wasserstoff und Stickstoff zeigen keine Abweichungen: es wurden bei den früheren Analysen im Mittel 11,35\% $\mathrm{H}$ und 1,76\% $\mathrm{N}$ gefunden. Eine Erklärung dafür wissen wir zurzeit nicht zu geben, doch ist zu erwähnen, daß die höheren Kohlenstoffwerte besser zu der aus den Spaltungsprodukten abzuleitenden Formel des Cerebrons, welche $69,65 \%$ G verlangt, stimmen. Für die Prüfung unserer Substanzen auf Phosphor wurden von der Fraktion B 2,0738 $\mathrm{g}$ und von der Fraktion D 2,2067 g nach A. Neumann unter Benutzung der von Gregersen ${ }^{2}$ ) empfohlenen Modifikation (10 ccm Säuremischung) verascht. Nach Zufügen von Wasser, Ammonnitrat- und Ammonmolybdatlösung in den von Gregersen vorgeschriebenen Mengen entstand eine ganz minimale gelbe Abscheidung, welche etwa um das Zehnfache geringer war, als diejenige, welche unter ganz den gleichen Verhältnissen $0,000075 \mathrm{~g} P$ (als Natriumphosphat) hervorriefen. Die Substanzen sind also als phosphorfrei $\mathrm{zu}$ bezeichnen.

Die drei Fraktionen D schmolzen in Übereinstimmung mit den früher für das Cerebron gemachten Angaben bei 212-213 ${ }^{\circ}$ zu einer klaren gelblichen Flüssigkeit. Die Fraktionen A und B zeigten annähernd den gleichen Schmelzpunkt.

1) Diese Zeitschrift, Bd. XLIV, S. 370.

2) Diese Zeitschrift, Bd. LIII, S. 453. 
470 Hermann Loening u. H. Thierfelder, Über das Cerebron. IV.

Bei der Hydrolyse der amorphen Substanz entsteht auch ein reduzierender Körper.

Die bis jetzt festgestellten Unterschiede zwischen dem Cerebron und der amorphen Substanz betreffen also nur die Löslichkeit und das Verhalten beim Erhitzen mit schwefelsäurehaltigem Methylalkohol. Bei der weiteren Untersuchung wird sich zeigen, ob es sich in der Tat um einen einheitlichen, dem Cerebron isomeren Körper handelt oder nur um ein mit einer Beimengung behaftetes Cerebron. ${ }^{1}$ )

Jedenfalls ergibt sich aus dieser Ârbeit, daß dem Cerebron hartnäckig eine andere Substanz anhaftet und daß auch ein schön krystallisiertes Präparat noch nicht ohne weiteres als rein angesehen werden darf. Wir müssen es auf Grund dieser Feststellungen für nicht unwahrscheinlich halten, daß die bei der Untersuchung der Spaltungsprodukte des Cerebrons hervorgetretenen Unstimmigkeiten auf die Verwendung nicht ganz reiner Präparate zurückzuführen sind. In der Tat findet sich in einer früheren Mitteilung die Angabe, daß die Lösung des Cerebrons in schwefelsäurehaltigem Methylalkohol bei 3-4stündigem Kochen einen leicht gelblichen Farbenton angenommen habe. Wir werden die Spaltungen mit sorgfältig gereinigtem Cerebron wieder aufnehmen.

In der Literatur finden sich schon Angaben über das Vorkommen eines zweiten Cerebrosids im Gehirn (Thudichum, ${ }^{2}$ ) Parkus, ${ }^{3}$ ) Kossel und Freytag. ${ }^{4}$ ) Der eine von uns (L.) ist damit beschäftigt, diese Körper nach den Angaben der Autoren herzustellen und untereinander und mit dem von uns erhaltenen $\mathrm{zu}$ vergleichen.

1) Wir haben auch an die Möglichkeit gedacht, daß der krystallisierende Körper erst während der Darstellung aus dem amorphen entsteht, etwa durch Eintritt von Methylgruppen. Die Experimente sprechen aber gegen diese Annahme.

2) Die chemische Konstitution des Gehirns usw. Tübingen 1901, S. 214 .

$\left.{ }^{3}\right)$ Journ. f. prakt. Chem. N. F. Bd. XXIV, S. 310.

$\left.{ }^{4}\right)$ Diese Zeitschrift, Bd. XVII, S. 431. 